\title{
Abstracts of \\ Canadian Meeting of neuro-oncology Réunion canadienne de neuro-oncologie
}

\author{
June 5-6, 1986 \\ Montreal, Quebec \\ Organizing Committee: C. Freeman \\ D. Stewart \\ N. Durepos, secretary \\ J.-G. Villemure, chairman
}

1.

Angiogenesis in Human Brain Tumours: Inhibition by Copper Depletion

STEVEN BREM (Montreal, Quebec)

The development of blood supply is a crucial step for the growth of solid tumours. Capillary endothelial proliferation is especially prominent in intracranial tumours. These tumours are a potent source of angiogenic factors. Recently copper ion has been shown to be an essential co-factor and possible regulator of angiogenesis.

To test the hypothesis that copper depletion could inhibit angiogenesis in vivo, we tested 425 tumour fragments from 10 patients harboring a spectrum of CNS neoplasms: astrocytoma ( 141 fragments), meningioma (76), acoustic schwannoma (75), medulloblastoma (47), choroid plexus papilloma (42), hemangioblastoma (44). Test fragments (208) were implanted in the corneas of rabbits placed on copper-deficient diets, supplemented with penicillamine, and 217 fragments were transplanted to normocopremic animals.

All ten CNS neoplasms stimulated a brisk neovascular response, regardless of histiotype. With reduction of the copper level; we observed that: 1) Angiogenesis was totally inhibited and failed to appear in $50 \%$ of the test fragments, as contrasted to $26 \%$ of the controls $(P<.025), 2)$ In the remaining eyes with an agniogenic response, there was a) Reduction in the number of average vessels/cornea (test $=53$, control $=70$ ) b) Delay in the onset of vascularization after implantation (test $=5$ days, control $=4$ days). c) Reduction in the rate of vascular growth (test $=0.12 \mathrm{~mm} / \mathrm{day}$, control $=0.16 \mathrm{~mm} / \mathrm{day}, \mathrm{p}<.025$ ).

It has been previously shown that inhibition of angiogenesis leads to the regression of experimental tumours. Our results indicate that copper depletion retards neovascularization induced by human brain tumours. By interfering with tumour angiogenesis, depletion of copper ion could serve as a new approach for the treatment of patients with human brain tumours.

2.

Vasogenic Edema in a Rat Glioma Model

\section{C.L. FARRELL and R.F. DEL MAESTRO (London, Ontario)}

Vasogenic brain edema associated with brain tumours affects brain mass and metabolism and is a critical determinant of morbidity in patients with intracranial tumours. The $\mathrm{C} 6$ spheroid implantation glioma model is suitable for the investigation of edema because it is a simple and reproducible rat model with pathological similarities to human glial neoplasms. The sites of production and distribution of edema were investigated in this experimental intracranial tumour. Edema is characterized by the extravasation of serum proteins. Evans blue (EB) which binds to serum albumin was measured spectrophotometrically in normal brain, tumour and peritumour areas in rats implanted with spheroids to quantitate vascular permeability during tumour development. Leakage of EB increased as tumours increased in size and then plateaued as necrotic centres developed. Fluoresceinconjugated antibodies against serum rat albumin were used to immunocytochemically localize the regional edema distribution on intact brain slices, and to simulate the mechanism of protein leakage spread. In the viable regions of tumour tissue, primarily in the periphery in large tumours, there was abundant fluorescence. There was some fluorescence in the peritumour areas and little or none in the central, necrotic portions of the tumour. Around individual vessels in the viable tumour tissue, a decreasing gradient of intensity of fluorescence from vessel to surrounding tumour tissue was observed. In the peritumour tissue, less than $10 \%$ of the vessels also demonstrated this pattern. Vessels in the contralateral hemisphere were negative. These results suggest that edema arises predominantly from vessels within the viable tumour mass and that there is a small population of vessels in the peritumour regions which appear to contribute. The spectrophotometric EB data and quantitative morphometry studies of the blood-brain barrier characteristics in these tissues are consistent with this conclusion.

\section{3.}

Endothelial Isolated from Malignant Cerebral Tumours: In Vitro Analysis

P. COSTELLO, E. STROUDE, E.J. WILSON and R.F. DEL MAESTRO (London, Ontario)

Endothelial cells from normal brain and tumour tissues are morphologically and functionally different. Endothelia have been isolated from several animal species, and human cerebral non-neoplastic tissue. In our laboratory, endothelium have been isolated from glial tumours obtained at the time of surgical resection. These human endothelium have been maintained in vitro to examine cell characteristics and angiogenesis at the cellular level. Microvessels were isolated using a homogenization method previously described (Debault and Cancilla. Adv Exp Med Biol 131: 69, 1980) and incubated at $37^{\circ} \mathrm{C}$ in modified 
Lewis media with $15-20 \%$ fetal bovine serum. One cell line, isolated from a glioblastoma multiforme using cloning ring techniques, grew in a "cobblestone" pattern. This cell line was positive for Factor VIII antigen, negative for GFAP staining and positive for Isolectin B4. In early passages, chromosome analysis demonstrated only mild abnormalities. These cells, designated as endothelium, have a longer doubling time than those isolated from non-neoplastic tissue. After only 10 passages ( 6 months), the cell line spontaneously transformed to reveal gross morphological and chromosomal abnormalities, but still stained positively for Factor VIII antigen. In early passage ( $p$ 3-6), the endothelial cells did not spontaneously form spheroids in spinner flask culture, but later passages formed spheroids of up to $500 \mu \mathrm{M}$ suggesting further alterations in the endothelial properties with increasing passage number.

\section{4.}

Nonsteroidal Anti-Inflammatory Drugs and Peritumoural Cerebral Edema

R.F. DEL MAESTRO, H. REICHMAN and C.L. FARRELL (London, Ontario)

Cerebral edema associated with brain tumours is experimentally and clinically decreased by corticosteroid therapy. Non-neural inflammatory processes are commonly treated with nonsteroidal anti-inflammatory drugs (NSAIDs). However, the influence of these compounds on cerebral edema induced by cerebral tumours has not been assessed.

The influence of two corticosteroids (methylprednisolone and dexamethasone) and two nonsteroidal anti-inflammatory drugs (indomethacin and ibuprofen) were studied using the rat $\mathrm{C} 6$ spheroid implantation model comparing protein extravasation quantitatively. Serum albumin extravasation was assessed using Evans blue dye which was measured in tumour, peritumour and contralateral tissue after intravenous injection.

Tumour using Evans blue extravasation was decreased in all treatment groups when compared to controls and Evans blue extravasation also decreased in peritumoural and contralateral brain.

The results demonstrate that tumour induced cerebral edema is decreased by both steroid and nonsteroidal anti-inflammatory drugs. The role of nonsteroidal anti-inflammatory drugs in human peritumoural edema is presently being investigated.

\section{5.}

The Effect of Diphenylhydantoin and Dexamethasone on C6 Astrocytoma Radiosensitivity

\section{LORDO, E. STROUDE and R.F. DEL MAESTRO (London, Ontario)}

Diphenylhydantoin and dexamethasone are commonly used in the management of patients with primary and secondary intracerebral neoplasms. The prophylactic use of diphenylhydantoin has been suggested for a number of intracerebral tumours. Patients with malignant glial tumours being treated with diphenylhydantoin and/or dexamethasone may undergo irradiation therapy as a part of their treatment. The influence of these compounds on the radiosensitivity of malignant astrocytoma cells is unknown. These studies have been carried out to assess the effects of clinical concentrations of diphenylhydantoin (20 $\mu \mathrm{g} / \mathrm{ml})$ and dexamethasone $(10 \mu \mathrm{g} / \mathrm{ml})$ on the growth and irradiation sensitivity of $\mathrm{C} 6$ astrocytoma cells in both monolayer and three dimensional spheroid culture.

Diphenylhydantoin significantly prolonged the doubling time (23\%) of $\mathrm{C} 6$ astrocytoma cells in monolayer culture but no significant changes were seen in spheroid growth or the plating efficiencies of cells obtained from spheroids at this concentration.

Dexamethasone also significantly prolonged doubling time (13\%) of C6 astrocytoma cells in monolayer culture. A similar decrease was also seen in three dimensional spheroid culture. Neither diphenylhydantoin
(20 $\mu \mathrm{g} / \mathrm{ml})$ nor dexamethasone $(10 \mu \mathrm{g} / \mathrm{ml})$ were associated with a significant alteration in irradiation sensitivity of $\mathrm{C} 6$ astrocytoma monolayer or spheroidal culture.

At the concentrations tested, both of these compounds significantly prolonged doubling times of $\mathrm{C} 6$ astrocytoma cells. However, they did not influence the irradiation sensitivity of these cells.

6.

Preliminary Characterization of a Human Monoclona Antibody (NEA-1) from a Patient with a Primitive Neuroectodermal Tumour

M.D. DAN and G.B. PRICE (Montreal, Quebec)

To test the hypothesis that brain tumour patients possess circulating B-lymphocytes reactive with autologous tumour, we performed a cell fusion between peripheral blood lymphocytes from a patient with a primitive neuroectodermal tumour and the human myeloma-like line TMH2 SP2. Hybridomas were grown in standard HAT medium for 1 week, then switched to HT and exchange-fed at least 4 times prior to screening. A sample of autologous tumour was obtained at the time of craniotomy, and a crude $3 M \mathrm{KCL}$ extract prepared. Standard polyvinyl chloride microELISA plates were coated with the extract, and the hybridomas screened for reactivity to autologous tumour using alkaline phosphatase-linked goat anti-human $k$ and $\mathrm{I}$. One hybridoma, NEA-1, reacted with $>$ mean +2 S.D. than control non-specific human immunoglobulin, and was selected for further testing.

NEA-1 was screened for reactivity against a number of $3 \mathrm{M} \mathrm{KCl}$ tumour extracts from different patients, and reacted with $\mathrm{p}<0.025$ with the following: $2 / 2$ glioblastoma multiforme, $1 / 2$ high grade astrocytomas, 1/1 metastatic poorly differentiated carcinomas of maxillary sinus origin, and $1 / 3$ pilocytic astrocytomas. NEA- 1 did not react with any of the following: $1 / 1$ oligodendrogliomas, $1 / 1$ meningiomas, $2 / 2$ low grade astrocytomas, and $2 / 3$ pilocytic astrocytomas. The association with high grade, anaplastic tumours was $\mathrm{p}<0.025$ by Fischer's exact test. In addition, NEA-1 was screened against a $3 \mathrm{M} \mathrm{KCl}$ extract of "normal" human brain obtained from a patient who was undergoing a craniotomy for seizures, and found not to cross-react. Using a modification of the above microELISA assay, two human glioma cell lines, SK-MG-1 and LN-340, were lightly fixed in $0.1 \%$ gluteraldehyde for 3 min then coated to plastic plates using poly-l-lysine. NEA-1 was found to bind strongly $(p<0.005$ ) to the line SK-MG-1 but not LN-340. An attempt to demonstrate binding to either of these two lines using the FACS was unsuccessful.

From these results it is clear that the antigen recognized by NEA-1 is restricted to the more anaplastic of the tumours so far tested, but is distributed among those tumours of ectodermal origin. The inability to demonstrate binding by FACS may indicate an intracellular localization of the antigen, since gluteraldehyde fixation of the line SK-MG-1 may expose such antigens. Further characterization of NEA-1 (e.g. immunoglobulin isotyping, level of production, DNA histogram, etc.) must await subcloning of the hybridoma.

7.

Metabolic and Hemodynamic Studies of Gliomas Using Positron Emission Tomography

J.L. TYLER, J-G. VILLEMURE, M. DIKSIC, A.C. EVANS, Y.L. YAMAMOTO and W. FEINDEL (Montreal, Quebec)

Positron emission tomography was used to evaluate 16 patients suspected of having high grade cerebral gliomas. All patients were studied before any intervention was undertaken. Measurements of cerebral glucose and oxygen metabolism, oxygen extraction, and blood flow and volume were obtained in all patients. In addition, $\mathrm{pH}$ measurements were obtained in 6 cases. Fifteen of these patients were later proven by biopsy to have gliomas, one had an intracerebral abscess. Of the 15 gliomas, 3 were Grade II, one was Grade III, and 12 were Grade IV. 
Compared to a homologous region in the contralateral hemisphere, tumour tissue demonstrated decreased oxygen extraction and oxygen metabolism, and increased blood volume. Compared to Grade II tumours, Grade IV tumours demonstrated higher relative oxygen extraction and utilization, and higher blood volumes. Tumour blood flow was quite variable, but tended to be higher in the higher grade tumours. Measurements of glucose utilization in tumour, using individually calculated rate constants, were variable, and did not consistently correlate with tumour size or tumour grade. These findings differ from results obtained from studies of gliomas after radiation and/or chemotherapy. ${ }^{1-2}$ Parietal tumours $(\mathrm{N}=6)$ tended to have higher relative glucose utilization and blood flow, and lower relative oxygen extraction, when compared to frontal tumours $(\mathrm{N}=4)$. Tumour $\mathrm{pH}$ differed significantly from the $\mathrm{pH}$ in contralateral brain (p .005); despite uncoupling of oxygen and glucose metabolism consistent with anaerobic glycolysis, alkalotic $\mathrm{pH}$ values were consistently seen in high grade tumours. Primary cerebral gliomas and surrounding cerebral tissue studied before intervention are different metabolically and hemodynamically from similar tissues after exposure to radiation or chemotherapy.

1. DiChiro G, Brooks RA, Patronas NJ et al. Issues in the in vivo measurement of glucose metabolism of human central nervous system tumours. Ann Neurol 15 (Suppl): S138-S146, 1984.

2. Patronas NJ, DiChiro G, Kufta $\mathrm{C}$ et al. Prediction of survival in glioma patients by means of positron emission tomography. J Neurosurg 62: 816-822, 1985.

This research was supported by NIH and MRC grants.

8.

\section{Measurement of the Metabolism of Primary Gliomas with Pet}

M. DIKSIC, J. TYLER, A.C. EVANS, M. KIRIKAE, E. MEYER, Y.L. YAMAMOTO, J.-G. VILLEMURE and W. FEINDEL (Montreal, Quebec)

The relationship between supply (blood flow) and utilization of nutrients to brain tumours must be determined before the tumour metabolic state can be evaluated. The possibility of monitoring in vivo changes in this interrelationship during tumour therapy could increase the effectiveness of treatment.

The rate constants for 2-FDG $\left(k_{1}{ }^{*}\right.$ to $\left.k_{3}{ }^{*}\right)$, glucose and oxygen utilization, blood flow, and blood volume in 12 patients with malignant gliomas were measured before treatment. Areas of very low radioactivity indicating tissue necrosis were not used for regions of interest. In a four-variable model, rate constants $\left(k_{1}{ }^{*}, k_{2}{ }^{*}\right.$, and $\left.k_{3}{ }^{*}\right)$ and blood volume were fitted to tissue curves during the first $40 \mathrm{~min}$ after a prolonged bolus ( $2 \mathrm{~min}$ ) injection of F-18 2-fluorodeoxyglucose. Rate constants in the tumour were generally different from those in contralateral brain. However, the ratio of $\mathrm{k}_{3}{ }^{*} / \mathrm{k}_{2}{ }^{*}$ (related to the lumped constant stability) is similar in the tumour and in contralateral gray matter. (Averages were 0.30 in tumour and 0.33 in gray matter.) Two patients had ratios significantly higher $(0.48, p<0.01)$ and lower $(0.21, p<0.05)$ than the mean level.

Regional blood flow, blood volume, oxygen extraction, and oxygen utilization were measured by an equilibrium method using $0-15$ labelled gases. Estimates for glucose utilization in the tumour were higher when regional rate constants were used than when rate constants were measured in normal volunteers. However, glucose utilization in tumour, calculated with regional constants, did not differ significantly from that measured with average rate constants. Our findings showed no significant difference between the blood volume in the viable part of the tumour and that in contralateral gray matter. Our limited number of cases revealed that the glucose and oxygen utilization, oxygen extraction fraction, and blood flow are lower $(p<0.05)$ in untreated malignant gliomas than in contralateral gray matter. These findings for newly diagnosed tumours are different from those reported by DiChiro et al for recurrent malignant gliomas.
This work has been supported in part by the Medical Research Council of Canada (SP-5), an N1H grant (No. 1-R01-NS-22230-01. NENA-T), and the Killam Memorial Scholarship Fund of the MNI.

9.

Rat Intracranial Tumour pH Modification In Vivo by Hyperglycemia

E.J. DOLAN, G. MIES, S. KRAJEWSKI, W. WECHSLER and K.-A. HOSSMANN (Toronto, Ontario)

Tumour $\mathrm{pH}$ can substantially affect both responsiveness to chemotherapy' and hyperthermia ${ }^{2}$ treatment in vitro. Little is known regarding brain tumour $\mathrm{pH}$, and no work has been done to try to alter brain tumour $\mathrm{pH}$ in vivo. We report preliminary results of the effects of hyperglycemia on brain tumour $\mathrm{pH}$ in vivo.

Intracranial F98 (gliosarcoma) tumours were stereotactically induced by cell suspension injection into the right caudate nucleus of rats. Seven to 10 days later, when symptomatic, the animals were anaesthetized with $0.8 \%$ halothane and randomly assigned into the control or treatment group. Controls were only monitored while the treatment group received a bolus of $0.5 \mathrm{ml}$ of $20 \%$ glucose over 45 seconds, followed by a continuous infusion to maintain plasma glucose over 30 $\mathrm{mM}$. Arterial blood gases were frequently monitored for both groups and 3 hours after the start of the infusion (or the monitoring), the brains were frozen in situ. After removal, $20 \mu$ frozen sections were placed on umbelliferone paper and illuminated at $340 \mathrm{~nm}$ and $370 \mathrm{~nm}$. and the resultant fluorescence recorded. Regional brain $\mathrm{pH}$ could then be obtained as described by Csiba et al. ${ }^{3}$

The 5 control animals developed 3 intracerebral and 3 subarachnoid tumours, while the 9 hyperglycemic animals developed 8 intracerebral and 4 subarachnoid tumours. Intracerebral tumours had alkaline $\mathrm{pH}$ 's and no significant difference existed between controls $(\mathrm{pH}=7.315$ \pm 0.074 SEM; $N=3$ ) and hyperglycemics $(\mathrm{pH}=7.281 \pm$ 0.056 SEM; $N=8$ ). While parts of the subarachnoid tumours showed similar $\mathrm{pH}$ 's to those of intracerebral tumours, there were marked differences between the two groups. In the control animals, $2.4 \%$. $6.1 \%$, and $4.1 \%$ of the subarachnoid tumour had a $\mathrm{pH}$ of 6.0 or less. while in the hyperglycemic animals $19.5 \%, 27.2 \%, 38.6 \%$, and $74.2 \%$ (p $<0.05$ ) of the tumour had $\mathrm{pH}<6.0$. Necrosis was not yet evident in the tumours with extensive low $\mathrm{pH}$ areas suggesting an acute decrease in $\mathrm{pH}$.

Our study has shown that subarachnoid tumour pH can be substantially lowered in a significant portion of the tumour. The factors influencing this effect are presently under investigation. and it remains to be determined if agents other than glucose can more effectively lower tumour $\mathrm{pH}$, particularly in intracerebral tumours.

1. Kennedy, K.A., McGurl, J.D., Leondaridis, L., Alabaster, O. Cancer Res. 45: 3541-3547, 1985.

2. Hofer, K.G., Mivechi, N.F. J. Natl. Cancer Instit. 65: 621-625. 1980.

3. Csiba, L., Paschen, W., Hossmann, K.-A. Brain Res. 289: 334-337, 1983.

10.

\section{Hypoxic Cell Cytotoxins: Potential for Glioblastoma Therapy}

D.J. CHAPLIN*. B.D. ACKER and C.M. LUDGATE (Vancouver, British Columbia)

The basis of selective toxicity is to exploit differences that exist between the normal and "invading" cell population. In the case of glioblastoma, one of these differences is that tumours have a lower oxygenation status than normal tissues, thereby protecting the tumour from radiation therapy. There is now evidence that hypotensive drugs can (by selectively reducing tumour blood flow) further compromise the oxygen status of malignant tissue. 
The present studies were designed to evaluate if hypotensive agents could increase the therapeutic efficiency of drugs known to be selectively toxic to hypoxic cells. Initial investigations have involved the use of the hypotensive drug hydralazine in combination with the "alkylating nitroimidazole" RSU-1069. The results obtained clearly indicate that hydralazine can potentiate the effects of RSU $=1069$ in the Lewis lung carcinoma when administered orally at a dose of $5 \mathrm{mg} / \mathrm{kg}$. However, this dose of hydralazine has no effect on the LD50/7d of RSU $=1069$ $(0.19 \mathrm{mg} / \mathrm{g})$ in C57BL mice. These initial results indicate that such an approach may have a role in cancer therapy. Further studies are now underway and will be reported, including the effects of hypotension on blood flow in glioblastoma.

*D. J. Chaplin, Ph.D., Staff Radiobiologist, Cancer Research Centre, 601 West 10th, Vancouver, B.C. V5Z IL3

11.

The Biology of Astrocytoma: Lessons Learned from Chronic Myelogenous Leukemia - Hypothesis.

\section{J. G. CAIRNCROSS (London, Ontario)}

Chronic myelogenous leukemia (CML) is an example of a "welldifferentiated" neoplasm that develops following neoplastic transformation of a precursor cell. The biology of astrocytic neoplasms can be interpreted in light of concepts that have emerged from studies of the biology of CML. I propose that astrocytomas arise from a pluripotential precursor cell whose progeny, although transformed, retain the ability to differentiate, and do so along astrocytic lines. The result is a neoplasm composed of "mature" tumour cells, similar one to another, and resembling normal astrocytes. Malignant change, like blast crisis in $\mathrm{CML}$, then occurs as a consequence of further molecular genetic events leading to accelerated growth and maturation arrest in a previously differentiating neoplastic cell. This hypothesis challenges the conventional view that astrocytomas arise from astrocytes and that malignant change occurs as a result of dedifferentiation.

12.

Neurosurgical Treatment of Intradural Extramedullary Spinal Metastasis

\section{R.G. PERRIN (Toronto, Ontario)}

Intradural extramedullary spinal metastasis is infrequently encountered and rarely reported. We have managed 10 such cases among 200 consecutive patients with symptomatic spinal metastasis treated in the Division of Neurosurgery, Wellesley Hospital, Toronto.

This series comprised four men and six women ranging in age from 39-68 years (mean 55 years). The culpable primary sites were breast in four cases, lung in three, melanoma in two, and uterus in one patient. The initial symptom in nine patients was pain, with five patients reporting a characteristically severe cramping discomfort with radicular distribution. All patients were treated with surgical decompression and intradural exploration. At the time of surgery, six patients were weak but ambulatory and four were bedridden. Following surgery, four patients enjoyed some measure of pain relief, seven became ambulatory, and three remained bed ridden. Two patients achieved a "satisfactory result" - walking and continent six months following surgery.

Secondary brain tumours were demonstrated or implicated in nine patients. It is thought this intradural extramedullary spinal metastases represent tertiary deposits following dissemination via the CSF from existing secondary sites.

Symptomatic intradural extramedullary spinal metastases cause a virulent clinical syndrome with poor prognosis and disappointing outcome after treatment. Given the high incidence of associated cerebral metastatic involvement, total neuraxis radiation and/or chemotherapy should be considered when symptomatic spinal metastasis is discovered to be intradural and extramedullary.

13.

Intra-Tumoural Chronic Chemotherapy of Malignant Cerebral Tumour by Stereotactic Approach

G. BOUVIER, R. PENN, J. CROIN, R.A. BÉIQUE, L. CLOUTIER, J. JOLIVET and J.G. BLAIN (Montreal, Quebec)

Serious efforts are made in order to find new forms of treatment for malignant brain tumour.

Experimental work done by Penn and Croin has proven that survival of rats with experimental malignant brain tumour has significantly increased using direct intra-tumoural infusion of cysplatinum.

Our experience with chronic depth electrode recording has proven that it is a reliable and safe technique. We have developed catheters for chronic infusion of chemotherapeutic agents.

We are presently reporting a case of malignant recurrent astrocytoma. This patient was previously operated in March 1982. A course of 5,500 rads of radiotherapy followed. The patient was functional until October 1984 when recurrence required surgery. It was followed by a systemic chemotherapy with AZQ with a dose of $5.5 \mathrm{mg}$ per square metre per day for 5 days. Immediate post-op result was excellent. 12 months later there was a marked increase in speech deficit which corresponds to a recurrence of the tumour. It is then that with the full consent of the patient and his family, that intro-tumoural chemotherapy was performed on August 19, 1985.

Following tumoural chemotherapy, no deterioration, no side effects were perceived. Neurological examination remained exactly the same that is severe dysphasia. Blood tests were all normal. No bone marrow depression. No sign of toxicity.

The method and equipment will be presented demonstrating the feasibility of delivering high concentration of chemotherapeutic agent directly into a brain tumour.

14.

Radiofrequency Hyperthermia for Malignant Brain Tumours: An Experimental Model

\section{BERNSTEIN, L. HEINZL and J.W. HUNT (Toronto, Ontario)}

In order to study the effects of heat alone, or in combination with other treatment modalities, the authors have designed an experimental system to study therapeutic hyperthermia in a small animal brain tumour model.

The experimental set-up consists of: 1) $\sim 6$ mm-diameter 9L tumours grown in the brains of F-344 rats by stereotactic inoculation of cells from in vitro culture; 2) an external radiofrequency (RF) inductive applicator which provides a large $R F$ current in a $1 \mathrm{~cm}^{2}$ conductor parallel to the rat's scalp; 3 ) a surface cooling system in which deionized water is circulated over the applicator enclosed in a water-tight chamber separated from the rat's scalp by a silicon membrane; 4) invasive thermometry consisting of $\sim 40 \mu$-diameter chromelalumel thermocouples supported by 5-0 silk sutures, which can be scanned mechanically across the brain and tumour to give a temperature profile.

Experiments with conducting gel phantoms indicate that deep heating to therapeutic temperatures is feasible. Studies in rats with intact skulls indicate that satisfactory spatial distribution of heating to potentially tumouricidal temperatures $\left(>42^{\circ} \mathrm{C}\right) 6 \mathrm{~mm}$ deep in the brain can be achieved with adequate surface cooling. However, the limited number of temperature measurements in relation to the whole tumour volume renders it impossible to document sufficient cytotoxic heating to the entire tumour. This remains a problem inherent in all hyperthermia systems. 
15.

Monitoring of Sensory Evoked Potentials During Surgery of Brain Tumours

\section{F. GENTILI, H. GHATE and C. CORRADO (Toronto, Ontario)}

Despite advances in instrumentation, the use of laser technology, and microsurgical techniques, neurosurgical procedures on tumours involving eloquent areas of the brain still carry significant risk for neurological injury. The use of intraoperative recording of sensory evoked potentials (SEP) has been advocated in monitoring neurological function during major neurosurgical procedures to reduce the risk of injury to neural structures.

The purpose of the present study was to determine the value of continuous intraoperative SEP monitoring as a technique for the early detection of impending neurological dysfunction and as a prognosticator for postoperative neurological deficit.

SEPs have been recorded during intracranial tumour surgery in 55 patients (40 supratentorial tumours; 15 infratentorial-basal tumours). While in the majority of patients with supratentorial lesions, somatosensory evoked potentials (SSEP) alone were monitored, patients with posterior fossa or basal tumours had both SSEP and brainstem auditory evoked potentials (BAEP) recorded. Some alteration in intraoperative SSEP parameters were noted in the majority of patients. Minor variations in amplitude and latency were most often related to changes in anaesthetic concentration or alteration in cardiovascular and respiratory parameters such as blood pressure, arterial blood gases, and temperature. Significant alterations in SEP parameters from anaesthetic baseline were seen in 11 patients. In 3 patients undergoing posterior fossa surgery, specific action was taken by the surgeon in response to SEP alterations with subsequent improvement in conduction. Persistent alteration in both BAEP and SSEP was associated with a postoperative brainstem dysfunction in 1 patient. No patient with a stable BAEP and SSEP at the end of the procedure suffered additional neurological deficit. In supratentorial lesions, 4 patients with stable SEP parameters awoke with significant postoperative deficits. Three of these patients had lesions localized to the motor cortex. Patients with significant and persistent alteration in SEP parameters that could not be related to changes in cardiorespiratory parameters had increased postoperative neurological deficits.

Based on our experience to date, we conclude that intraoperative SEP monitoring may be of value in detecting and minimizing neural injury during major neurosurgical procedures.

16.

Periaqueductal Tumour as a Cause of Late Onset Aqueductal Stenosis

\section{P. STEINBOK and M.C. BOYD (Vancouver, British Columbia)}

Localized periaqueductal tumours usually present as hydrocephalus thought to be related to late onset congenital aqueductal stenosis. Unfortunately, the pathological diagnosis has often been unsuspected until post-mortem examination revealed the presence of a tumour.

In the past, radiological investigations, including positive contrast ventriculography, pneumoencephalography and even contrast CT scanning have frequently failed to show tumours in this region in the early stages. However, recent experience using MRI on patients with unexplained late onset aqueductal stenosis has in some instances shown the presence of a localized periaqueductal tumour as the cause of obstruction of the aqueduct of Sylvius.

We discuss four patients who presented with hydrocephalus secondary to presumed late onset congenital aqueductal stenosis, all of whom were shown to have a focal periaqueductal tumour. One patient had been investigated with a pneumoencephalogram and positive contrast ventriculogram and a CT scan with an early generation scanner, but the tumour was diagnosed only at the time of autopsy. In a second patient, the tumour was diagnosed by $C T$ scan and also confirmed with an MRI scan, but no histologic diagnosis has been obtained up to this time. In two other patients, CT scans with and without contrast enhancement were negative and in one of these patients, a positive contrast ventriculogram was also felt to be negative, but the tumour was easily identified on MRI scans. In one of these patients, a histologic diagnosis was obtained initially by biopsy and later at autopsy, but the second patient has had no biopsy up to the present time.

Periaqueductal tumours must be considered in the differential diagnosis of patients who present with late onset aqueductal stenosis and in such patients, MRI would appear to be the investigation of choice.

17.

Management of Medulloblastoma in Children - Retrospect and Prospect

\section{J.E. BLUNDELL (Montreal, Quebec)}

The management of medulloblastoma in children has always been difficult and remains unsatisfactory. A personal review over the last 25 years is presented with particular reference to improvements in methods of investigation and surgical techniques, with reference to changing radio-therapy techniques and to the introduction of chemotherapeutic agents. A preliminary in vitro study of chemotherapeutic agents is presented, aimed to assess beneficial effects while minimizing the risk of side effects from inefficient agents used in vivo.

Survival rates are reviewed, both from this series and from other published studies, with particular reference to sex and age of onset. Longevity is commented on, but with greater emphasis on the quality and normal enjoyment of life than on the mere number of years survived since diagnosis and treatment.

Since the original description of the medulloblastoma by Bailey and Cushing, and Bailey's subsequent regret at his inability to identify a medulloblast, much effort has been given to a clearer understanding of the histology of this tumour, which will be discussed in the next paper.

\section{8.}

Medulloblastoma with Favourable Outcome: Retrospective Pathological and Immunocytochemical Delineation of Characteristic Features

\section{K. MEAGHER-VILLEMURE and J.-P. DE CHADARÉVIAN (Montreal, Quebec)}

The prognosis of Medulloblastoma is regarded as poor. The unexpected good results in 12 of the last 39 cases seen at the Montreal Children's Hospital prompted a retrospective study. Its goal was to identify features which would allow prediction of favourable outcome.

This study included review of clinical charts and pathological assessment with histological, ultrastructural and immunocytochemical evaulation of the tumours obtained from all patients who were tumour-free 3 to 25 years after surgery.

There were 7 boys and 5 girls in this group. The age ranged between 1 and 9 years at time of diagnosis. The tumour was located in the vermis in 9 cases. The other 3 were in the right cerebellar hemisphere.

Histologically, all tumours showed both, a lobulated pattern and a diffuse pattern made of sheets of parallel rows of cells. Variable amounts of Homer-Wright rosettes, mild reticulin content and moderate vascularization component were also present.

Immunoperoxidase studies for GFAP, NSE, NF and S100 protein demonstrated neuroblastic differentiation and minimal astrocytic participation. It showed scattered GFAP and S100 protein positive cells at the periphery of the lobules and in the septae, in all cases. However, NSE was strongly positive in many cells within the lobules. The degree of positivity was variable in the diffuse pattern areas. NF was weakly positive in the fibrils seen in the center of the rosettes and lobules. 
Ultrastructurally, in the 8 cases studied, large cells with neuroblastic features could be found. They had elongated cytoplasmic processes rich in microtubules. Moderate amounts of small dense membranebound neuro-secretory granules were seen in the cytoplasm in all cases. Two cases showed clear vesicles with one having well-developed synaptic complexes.

Evaluation of the age factor in the 39 patients reviewed showed that, under 4 years of age, the incidence of Medulloblastoma with favourable outcome approached $50 \%$. After 4 years of age, the patient's chance of having a favourable biological behaviour declined to $25 \%$.

It is concluded from the study that evidence of minimal glial participation and clear neuroblastic differentiation as demonstrated by electron microscopy and immunocytochemistry characterizes the Medulloblastoma with favourable prognosis, a tumour by far more prevalent in children under 4 years of age.

\section{9.}

\section{Primary Malignant Lymphoma of the Central Nervous System}

\section{C.R. FREEMAN, C. SHUSTIK, M-L. BRISSON, K. MEAGHER- VILLEMURE and I. DYLEWSKI (Montreal, Quebec)}

Between 1960 and 1983, nineteen patients with primary malignant lymphoma of the central nervous system were seen at McGill University Hospitals. The diagnosis was made at autopsy in three patients, and by biopsy in sixteen. Results of treatment were poor. All four patients who underwent surgery alone died within two months of diagnosis. Of twelve patients who underwent surgery and postoperative radiotherapy, eleven died between 2 and 56 months (median 12 months) following diagnosis, and one is alive with disease at 47 months. Patterns of involvement at first recurrence and/or at autopsy were analysed for 13 patients. Failure at the original site of involvement was unusual after treatment consisting of surgery and radiotherapy. In contrast, failure in the brain at sites other than those originally involved was common in spite of the use of whole brain irradiation. Local leptomeningeal involvement was seen in one patient whose diagnosis was made at autopsy, and CSF seeding was seen in two additional patients, one within one month of diagnosis and one at relapse at 6 months after diagnosis. No patient developed disease outside the CNS. The limitations of current therapy for this disease are discussed, and certain suggestions made regarding the management of future patients with this diagnosis.

20.

\section{Traitement et Evolution des Tumeurs Hypophysaires}

\section{A. GODON et G. LACHANCE (Montréal, Québec)}

La présente étude a porté sur tous les cas de tumeurs hypophysaires traitées dans les deux hôpitaux depuis 1969, sur une période de 17 ans (en fait toutes opérées entre 1972 et 1986).

60 patients ont subi une chirurgie hypophysaire, la plupart par voie trans-sphénoïdale (55: T.S.) et rarement sous-frontale (5: T.F.).

Parmi ceux-ci: 9 patients ont été réopérés pour des récidives (T.S.) dont 7 pour une récidive, et 2 pour 3 récidives successives.

Les âges variaient entre 19 et 75 ans, avec un âge moyen de 47 ans.

Nous avons étudié avec précision les taux de récidives des tumeurs, avec ou sans radiothérapie post-opératoire. Ainsi 23 patients sur $\mathbf{6 0}$, soit $38 \%$ ont subi de la radiothérapie, dont 11 d'emblée après la première exérèse tumorale et à cause d'extensions supra et infrasellaires, et 12 autres traités par radiothérapie maximale après une première récidive. 3 patients ont refusé la radiothérapie, malgré 3 récidives successives.

Dans les récidives, on note 7 cas d'adénomes chromophobes envahissants, 2 cas de tumeurs mixtes (chromo et acidophile, chromo plus $\mathrm{T}$, à prolactine) I cas de tumeur surrénalienne doublée d'une $\mathrm{T}$. Basophile, 1 prolactinome et 1 acidophile.

La radiothérapie n'a pas empêché les récivives multiples chez 3 malades sur 15 cas de tumeurs non sécrétantes. Contrairement au passé, de nos jours elle est de plus en plus utilisée comme traitement primaire associé à la chirurgie.

Elle s'est avérée très efficace dans la prévention des récidives dans les tumeurs sécrétantes: microadénomes à prolactine, tumeurs à T.S.H., T.R.H. etc.

Nous avons porté attention au problême de l'insuffisance hypophysaire et son aggravation possible par la chirurgie et la radiothérapie.

La radiothérapie n'a pas causé d'insuffisance mesurable par les dosages endocriniens. Dans les tumeurs sécrétantes, elle a permis de diminuer l'hyperfonction hypophysaire et aussi la médication de contrôle et même dans certains cas (2) de rétablir une fonction glandulaire normale. Dans tous les cas, nous avons noté l'absence de récidives. Les tumeurs non sécrétantes causent une insuffisance hypophysaire progressive par compression lente (la plupart des cas) ou rapide (apoplexie hypertensive intratumorale).

$37.9 \%$ des patients traités par chirurgie seulement ont présenté une auggentation de leur insuffisance hypophysaire ( 13 sur 37 traités ainsi): 7 cas où l'insuffisance a été aggravée, et 6 où elle est apparue après la chirurgie. Chez 24 autres, les données pré-opet post-op sont demeurées similaires $(25 \%=$ panhypopit; $75 \%=$ Hypopit $)$.

Chez 2 cas sur 23 traités par chirurgie et radiothérapie, l'insuffisance a augmenté légèrement, mais il s'agissait de tumeurs non sécrétantes envahissantes, avec complications importantes en post-op, et il est probable que le pan-hypopituitarisme se soit aggravé lentement et inexorablement, post-chirurgie et sans relation avec la radiothérapie.

Quant au traitement optimal des tumeurs, il s'oriente ainsi: remplacement hormonal et radiothérapie dans les microadénomes sécrétants; microchirurgie, remplacement hormonal et radiothérapie dans les tumeurs non sécrétantes.

21.

\section{Cerebral Gangliogliomas - Review of 24 Cases}

M.F. LEVESQUE, K. MEAGHER-VILLEMURE, F. DUBEAU, J.-G. VILLEMURE, D. MELANSON, F.L. QUESNEY, R. MOUMDJIAN, A. OLIVIER, W. FEINDEL, T. RASMUSSEN and G. BERTRAND (Montreal, Quebec)

Ganglioglioma is a rare tumour of the C.N.S. Three recent cases operated in 1985 led us to review the surgical experience at the MNI. The clinical, radiological, electrophysiological and pathological aspects of 24 cases are presented, which is the largest clinical series in the present literature. The age at the time of diagnosis varies from 7 to 66 years old, with a mean of 19.6 years and the cases are equally distributed between the sexes. The initial presentation was a focal or generalized seizure (19 cases) or an intracranial space-occupying lesion (5 cases). Ictal or interictal abnormalities are most often seen on serial EEGs. Since 1978, 18 cases had CT scans which showed characteristic cystic hypodensities which enhanced on infusion in 15 cases or were normal in 3 cases. Recently the MRI in three cases showed high intensity signals well delineated in the amygdala and uncus without clear mass effect; two of them had these signals centered on homogeneous hypo-intense signals interpreted as calcifications.

The location of these tumours was as follows: 15 were in the temporal lobe, 3 in the frontal lobe, 3 parietal, 1 occipital, 1 cerebellum and 1 in the pineal gland. One patient underwent stereotaxic biopsy, all the others underwent partial to total surgical excision. All cases showed proliferation of both ganglionic and glial elements (astrocytic or oligodendrocytic). Some cases received post-operative radiation therapy. The prognosis varies depending on the location of the tumour, the extent of the resection but is in general good. The longest follow-up reaches 17 years after surgery without any neurological deficit. 
22.

\section{Meningeal Carcinomatosis: Diagnosis, Therapy, Prognosis}

\section{J.G. BLAIN, M. BLAIS and T. VUONG (Montreal, Quebec)}

Forty-eight patients with a diagnosis of carcinomatous meningitis are reviewed. Eleven patients were rejected because of associated proven parenchymal metastases. Thirty patients had proven carcinomatous meningitis without evidence of cerebral metastases as demonstrated by negative CT scan or autopsy, 27 had primary breast neoplasms, 8 with lung neoplasms, 1 ovary-cystadenocarcinoma and one of unidentified origin.

The onset of symptoms occurred in less than 2 years after diagnosis of the primary carcinoma in $51 \%$ of patients, with a range varying from the meningitis as a presenting symptom in 3 patients to 23 years after the diagnosis of the primary neoplasm in one patient.

The survival after diagnosis varied from 6 days to 21 months. Death occurred in less than 2 months in more than $50 \%$ of patients. Three patients survived more than one year.

Two clinical syndromes could be defined:

1) A cerebral syndrome, seen in $78 \%$ of patients, characterized by headache usually of a vice-like nature followed by nausea and somewhat less frequently by vomitting preceeding the clinical signs of meningitis. Unsteadiness of gait was a frequent symptom followed by diplopia. These symptoms frequently preceeded by weeks and even months signs of meningeal irritation.

2) A radicular syndome; in its early stages it is less well defined, the patients presenting vague symptoms involving the lower limbs. When better established, there is weakness of the lower limbs associated with lower limb paresthesia. Ill-defined pain in the lumbo-sacral regions with thigh, buttock radiation can be found.

Untreated patients had a survival of less than 2 months, with 2 patients surviving for longer periods. Patients treated with intrathecal lumbar Methotrexate fared better with more than $65 \%$ of patients surviving more than 60 days.

Early detection appears imperative if a cure of this more frequently seen site of metastases is to be anticipated.

\section{3.}

Tumeurs Primaires du Poumon et du Colon-Sigmoide et Metastase Cérébrale avec Survie Prolongée

E. BERGER, C. AUGER, G. ChOLETTE, J.J. GAGNON et A. NEAGA (Montréal, Québec)

En 1978 une femme âgée de 63 ans subissait une lobectomie supérieure droite pour épithélioma malpighien mal différencié. (G.C.) Evolution satisfaisante. Un an plus tard une nouvelle hospitalisation à cause d'une hémiparesie droite progressive avec dysphasie. L'investigation neuro-radiologique a mis en évidence une lésion occupante de l'espace dans la région temporale gauche. Une craniotomie temporale gauche est faite avec exérèse d'une métastase d'un épithélioma épidermoìde d'origine pulmonaire. (E.B.) Radiothérapie post-opératoire. Excellente évolution avec récupération totale de la motricité et presque totale de la dysphasie. Aucun signe de récidive tumorale pendant six ans au niveau du cerveau. En 1985, signoïdectomie pour épithélioma glandulaire assez bien différencié du colon sigmoïde. (J.J.G.) Une tomodensitométrie axiale cérébrale fait en ce moment ne montre pas de récidive tumorale au niveau du cerveau.

Billroth fut le premier auteur à décrire des tumeurs primaires multiples. Des études récentes ont indiqué le pourcentage des tumeurs primaires multiples au niveau du trajet aero-digestif comme étant de $20 \%$. Une incidence plus élévée fut déjà rapportée dans certains autres séries. Notre cas se situe dans le groupe de carcinomes multi centriques métachroniques les lésions étant apparues à un intervalle plus grand que la période de six mois des carcinomes synchrones. Notre cas se concorde avec les critères histologiques tel que statués par Warren \& Gates, c.à.d. premièrement la néoplasie doit être maligne, deuxièmement elle doit être séparée géographicalement \& troisièmement que la deuxième néoplasie ne représente pas une métastase. Notre cas d'ailleurs présente un certain intérêt car il semble avoir eu une guérison de plus de six ans après resection de la lésion pulmonaire ainsi que de la lésion cérébrale.

\section{4.}

\section{Malignant Melanoma and the Central Nervous System}

\section{R.F. DEL MAESTRO and I. MENDEZ (London, Ontario)}

Six hundred and fifty-two patients with histologically proven malignant melanoma have been followed by the London Regional Cancer Centre from 1960 to 1984. Fifty-five patients (8.4\%) developed neurological signs and symptoms due to secondary metastases to the brain. Seventy-one percent were male and $29 \%$ were female; the mean age was 49 years. Multiple lesions were found in $61 \%$ and single metastasis in $39 \%$. The most common site for the primary lesion in females was the lower limb (37\%) while for males it was the trunk (44\%).

Five month survival of patients with a single metastasis was $58 \%$ if wide surgical excision was possible and $25 \%$ of these patients survived greater than two years. In patients with multiple metastases that received radotherapy, survival times of greater than 5 months were found in $12 \%$ of the patients.

Patients with single metastasis should be treated aggressively by radical surgical removal of the lesion. Postoperative radiotherapy should be considered in cases of recurrence in the same site of the initial metastasis. In patients with multiple metastases at the initial presentation, radiotherapy has little impact on survival and its effectiveness should be carefully assessed as a palliative method of treatment.

\section{5.}

Timing of Radiation Therapy in Supratentorial Grade 2 Astrocytomas in Adults

\section{W.S. TUCKER and E. KLODAS (Toronto, Ontario)}

The efficacy and safety of radiation therapy in the treatment of slow-growing glial tumours remains controversial.

Since January 1977, 62 supratentorial grade 2 astrocytomas in adults have been treated at one hospital. One surgeon has delayed radiotherapy until recurrence requiring a second resection (GROUP I), while 3 surgeons radiated all patients after the initial biopsy and/or resection (GROUP 2). The cases were reviewed retrospectively and compared. The GROUPS were comparable with regard to factors considered prognostically significant.

9 of 28 patients in GROUP 1 required surgery for recurrence after an average interval of 2.88 years. 13 of the 34 GROUP 2 patients developed a recurrence, at an average interval of 3.25 years. The radiation was similar in the 2 GROUPS. Cobalt 60 , via 2 ports, in $200 \mathrm{rad}$ fractions, over 5 weeks, 105,000 rads. At 5 years, $56.5 \%$ of the GROUP 1 patients at risk were alive, and $37.8 \%$ of the GROUP 2 patients at risk were alive. The functional status of patients was identical overall (average Kamovsky score $82.1 \%$ in each GROUP).

10 pairs of patients were matched from the 2 GROUPS for preoperative duration of symptoms, age at initial resection (by decade), and extent of initial resection. 8 of the 10 GROUP I patients are still living, compared to 6 of the 10 GROUP 2 patients.

While not statistically significant, these data suggest that delaying radiotherapy in these patients does not compromise the quality or duration of survival. 
26.

\section{A Phase I Study of Hyperfractionation in Brain Stem Gliomas in Children}

\section{FREEMAN, D. NORRIS, R. STANFORD, M. COHEN, P. BURGER and J. BOYETT (Montreal, Quebec)}

In 1984, the Pediatric Oncology Group initiated a phase I study to assess the feasibility. immediate and late side effects and response to treatment in patients with brain stem gliomas treated with twice daily radiotherapy, and to test the feasibility of dose escalation in this group of patients. Patients between the ages of 3 and 21 years with a previously untreated tumour in the mesencephalon, pons and medulla oblongata were eligible for study. Biopsy proof of diagnosis was considered preferable, but patients without histologic confirmation of diagnosis were eligible for study if the clinical and radiological findings were unequivocal.

Between September 1984 and December 1985, 34 patients were entered on study. Two deteriorated rapidly and did not complete radiotherapy. The remainder received $110 \mathrm{cGy}$ twice a day to a total dose of 6600 cGy over 6 weeks. Of the 32 patients completing treatment with radiothereapy, 15 responded. Ten remain well at intervals of 2 to 6 months (median 3 months) following initiation of treatment. Five recurred at the primary site at 4, 5, 6, 7 and 9 months. Six have stable disease at 2 to 14 months (median 4 months) and one, while having stable disease at the primary site at 9 months following initiation of treatment, developed metastatic disease in the spinal axis. Nine never responded to treatment, and developed clearly progressive disease at intervals from shortly following completion of treatment to 10 months (median 3 months). One patient was lost to follow up immediately following completion of treatment. Early toxicity has been minimal.

The preliminary results and plans regarding dose escalation will be discussed in detail.

\section{7.}

Results of Treatment of Glioblastoma Multiforme with Pimesons at Triumf

\section{G.B. GOODMAN, R.W. HARRISON, G.K.Y. LAM, C.M. LUDGATE} and M. POMEROY (Vancouver, British Columbia)

Treatment results for brain tumours at TRIUMF appear to be significantly better with the use of localised tumour therapy using Pimesons (pions) only. This presentation reviews results achieved in 44 patients together with prognostic factors.

28.

Anti-Platelet Therapy: A Way to Improve the Therapeutic Index in the Radiation of Brain Tumours

C.M. LUDGATE, D.J. CHAPLIN and G.B. GOODMAN (Vancouver, British Columbia)

Radiation therapy for brain tumours is limited by late toxicity resulting in ischemic necrosis. The neurons are not radiosensitive as they do not divide. Late damage is caused by damage to endothelial cells with resulting vascular occlusion. Animal studies have shown that late damage is reduced by twenty percent by anti-platelet drugs. This has been substantiated by clinical trials in abdominal radiation.

The rationale and results of therapy in brain tumour patients will be presented and the future possibilities discussed.
29.

Brachial Plexopathy in Patients with Breast Cancer

\section{D.S. FULTON (Edmonton, Alberta)}

Brachial plexopathy is a known complication of breast cancer, usually caused by metastatic tumour infiltration or radiation fibrosis. Fiftysix breast cancer patients referred for neurological evaluation because of arm symptoms such as pain, weakness, paraesthesias or sensory loss, were reviewed.

Definite metastatic plexopathy was diagnosed in 19 patients who had had no brachial plexus radiation treatment and had metastatic disease adjacent to the plexus (axillary or supraclavicular nodes, lung apex). Probable metastatic plexopathy was diagnosed in 8 patients who had had radiation treatment and also had known metastatic disease adjacent to the plexus. For patients with metastatic plexopathy, median interval from breast cancer diagnosis to plexopathy was 35 months (range 3-221), 18/27 patients had pain, 21/17 motor deficit, 22/27 sensory deficit, 5/27 Horner's syndrome, 17/27 lower plexus involvement, $4 / 27$ upper, and 6/27 both upper and lower plexus involvement.

Radiation-induced plexopathy was diagnosed in 4 patients in whom plexopathy was present for 2 years without evidence of metastatic tumour. Interval from completion of radiation therapy to plexopathy was 2-178 months, $2 / 4$ patients had pain, $3 / 4$ motor deficit, $4 / 4$ sensory deficit, $0 / 4$ Horner's syndrome, $2 / 4$ lower plexopathy and $2 / 4$ both upper and lower plexus involvement.

Reversible plexopathy occurred in 14 patients. All had had radiation therapy to the plexus. Median interval from radiation to plexopathy was 2.5 months, $3 / 14$ patients had pain, 1/14 motor deficit, 13/14 sensory deficit, $0 / 14$ Horner's syndrome, $12 / 14$ lower plexus involvement and $2 / 14$ both upper and lower plexus involvement. Complete spontaneous recovery occurred in 13/14 patients and $I$ had minor residual motor deficit.

The etiology of the plexopathy could not be determined in 11 patients.

Metastatic disease is the most important cause of brachial plexopathy in breast cancer patients. A prospective study is needed to determine factors which may influence the development of plexopathy and to determine how it may be prevented.

\section{0.}

Cancer Pain at Memorial Sloan-Kettering Cancer Centre (MSKCC) Clinical Characteristics and Outcome

D.E. MOULIN and K.M. FOLEY (London, Ontario and New York, New York)

The Pain Service at MSKCC evaluated 165 inpatients seen consecutively in consultation for pain between July and December, 1983. Patients were followed until death or until the end of the follow-up period - March, 1985. By the end of this follow-up period $80 \%$ of the patients had died. The mean survival in this patient group post-consult was 4.1 months.

Two-thirds of the patients had pain on the basis of tumour infiltration of bone or nerve. The remainder suffered from tumour infiltration of fiscera or soft tissue or developed a pain syndrome secondary to cancer therapy. The most common malignancies producing pain in this series were lung (18\%), urinary tract (13\%), gynecological system (12\%) and G.I. tract (12\%). In each of these tumours neuropathic pain (usually due to tumour infiltration of either the brachial or lumbosacral plexus) was more common than bone pain. Despite the fact that breast cancer is a common cause of cancer pain, it accounted for only $8 \%$ of pain consults and in almost all cases the underlying problem was widespread bone pain. 
A variety of analgesic drugs provided the mainstay of treatment. 43 of the 52 patients $(83 \%)$ with bone pain enjoyed excellent or moderate pain relief but only 36 of $58(62 \%)$ patients with neuropathic pain had the same success.

In cancer patients with advanced disease, tumours producing neuropathic pain are more likely to result in pain consultation than tumours producing bone pain. A corollary conclusion is that neuropathic pain is more difficult to control than bone pain. Deafferentation and ectopic impulse formation in damaged nerves are the likely mechanisms responsible for poorly controlled neuropathic pain.

31.

Developmental Outcomes Following Treatment for Acute Lymphocytic Leukemia (ALL)

\section{H.G. TAYLOR (Montreal, Quebec)}

Developmental and educational consequences of the treatment for acute lymphocytic leukemia (ALL) were examined in a group of 27 elementary school children who were survivors of the disease. Each ALL survivor was compared to one normal sibling on a comprehensive battery of tests, and on measures of behavior and school performance. The ALL group performed less well than their siblings on tests of IQ, neuropsychological skills, and academic achievement. According to ratings by parents and teachers, the ALL group were less able to meet the academic demands of school, but did not differ from their siblings in behavioral adjustment. A greater number of children from the ALL group obtained borderline to low-average full-scale IQ scores: more of them performed below the 25th percentile on tests of academic achievement; and more were receiving special educational assistance at school. However, none of these children met criteria for mental retardation. Higher doses of brain radiation used to prevent or treat CNS leukemia were associated with lower IQ scores, poorer neuropsychological performances and greater difficulties in school as judged by parent ratings and by special help received. The present findings help to clarify the nature and predictors of risks associated with treatment for ALL in a group of children on a variety of treatment protocols.

32.

Etude Retrospective des Tumeurs Intracraniennes en Estrie de 1970 à 1985: Proposition d'une Fiche Statistique Prospective

\section{A. NABID, D.P. LUNEAU et J.B. LAMARCHE (Sherbrooke, Québec)}

En Estrie, près de 600 patients ont été référés pour tumeurs intracrâniennes au CHU de Sherbrooke au cours des quinze dernières années.

L'étude rétrospective des dossiers, faite à l'aide d'une fiche statistique comportant 39 items, a permis de préciser des aspects cliniques, histopathologiques, paracliniques, thérapeutiques et évolutifs de ce groupe de patients.

Cet échantillon a été revu relativement à sa représentativité par rapport aux groupes déjà publiés.

L'étude critique des dossiers au cours de celte revue a débouché sur l'institution d'une fiche prospective standard qui devrait être ajoutée comme complément au dossier de cette catégorie de patients.

Un modèle discuté par les neurologues, neurochirurgiens, neuropathologistes, radiologistes, radiothérapeutes, chimiothérapeutes, archivistes et statisticiens est proposé avec justification des principaux items.
33.

Volume Assessment of Brain Tumours

A. GIRARD, L. GERIG, M. RIDING, D.J. STEWART, Z. GRAHOVAC. P. GENEST and M. Mahadivan (Ottawa, Ontario)

Adequate assessment of brain tumour evolution is important in determining a therapeutic approach and essential in the evaluation of new therapeutic modalities. This can be difficult, as clinical evaluation is only qualitative and radiological assessment of tumour volumes is imprecise due to the irregular shape of most tumours and the difficulty in resolving their boundaries. The use of closely spaced CT slices allows precise measurement of total tumour volume by employing a simple Simpson Rule integration. In addition to "total tumour". contrast enhanced volume and volume of oedema are also determined and might be important in differentiating between tumour and normal tissue.

To assess the validity of these measurements, we analyzed data from 13 patients treated by a permutation or combination of surgery, radiotherapy and chemotherapy. For each patient we collated the clinical evaluation from progress notes, initial radiological evaluation from radiological reports, a retrospective analysis of estimated tumour growth based on a review of CT scans ad a volumetric analysis of the various measurable entities described above. Comparison between these different types of assessment and the value of volumetric analysis will be presented.

34.

Investigation of the Transport of SarCNU, A New Chloroethylnitrosourea (CNU) with an Amino Acid Amide Carrier

\section{SKALSKI, L. PANASCI and W. FEINDEL (Montreal, Quebec)}

SarCNU is a chloroethylnitrosourea with a sarcosinamide (methyl glycinamide) carrier. Comparative studies of BCNU vs. SarCNU demonstrated that SarCNU is less myelotoxic than BCNU in the CFU.C assay. SarCNU also exhibited increased antitumour activity against primary human glioma cells in the human tumour cloning assay. as compared to BCNU. This suggests that SarCNU may be more active against human gliomas that $\mathrm{BCNU}$ at doses which are less myelosuppressive. In order to determine if differences in transport of the CNU's could account for the increased antitumour activity of SarCNU. the cytotoxicity of SarCNU and BCNU was evaluated in media plus or minus amino acids or sarcosinamide. If SarCNU is transported by an amino acid or amino acid amide (sarcosinamide) carrier, the presence of the amino acids or sarcosinamide should inhibit the uptake and thus the cytotoxicity of this agent. The transport of SarCNU into a human glioma cell line, SK-MG-I, was investigated by examining the cytotoxicity of SarCNU and BCNU in the presence and in the absence of amino acids. Amino acid deprivation did not change the cytotoxicity of either CNU, which suggests that it is unlikely that SarCNU is accommodated by an amino acid transport system (BCNU uptake is by passive diffusion). The existence of a sarcosinamide-specific transport for SarCNU in SK-MG-1 cells was examined by comparing the cytotoxicity of SarCNU with or without a large molar excess of sarcosinamide. BCNU was similarly studied to control for non-specific effects of sarcosinamide. The cytotoxicities of both BCNU and SarCNU were significantly diminished in the presence of sarcosinamide. In the case of BCNU, this effect appeared to result from the observed large increase in the halflife of BCNU in the presence of sarcosinamide. The half-life of SarCNU was only slightly prolonged by sarcosinamide, therefore, the decreased cytotoxicity of this $\mathrm{CNU}$ may indicate a sarcosinamide-specific transport. Investigations of transport utilizing ${ }^{14} \mathrm{C}$-SarCNU will clarify these results. 
35.

\section{Cerebral Herniation Complicating Cisplatin Chemotherapy}

J.G. CAIRNCROSS, R.W. WALKER and J.B. POSNER (London, Ontario; New York, New York)

Five patients with recurrent malignant brain tumours (2 GBM, 2 medulloblastoma, I carcinoma) herniated within 6 hours of cisplatin chemotherapy. Two patients had clinical evidence of raised intracranial pressure prior to treatment. Baseline CT scans revealed either solitary, laterally placed mass lesions with midline shift ( 2 patients) or multiple bihemispheric mass lesions with surrounding edema. All had a normal serum $\mathrm{Na}^{+}$prior to treatment. Treatment consisted of cisplatin $\left(100-150 \mathrm{mg} / \mathrm{m}^{2}\right)$ together with IV hydration ( $2 \mathrm{x}$ maintenance in 4 patients) and mannitol. Focal and generalized seizures heralded cerebral herniation in 2 patients. The patients with lateral solitary masses had clinical evidence of uncal herniation with a Kernohan's notch. Post-herniation CT scans showed increasing edema and mass effect in 2 patients and were unchanged in 2 others. All patients were hyponatremic $\left(\mathrm{Na}^{+}\right.$ 115-134) at the time of herniation. Two patients died without regaining consciousness, and both had autopsy evidence of herniation. The pathogenesis of rostral-caudal deterioration following cisplatin therapy appears to be multifactorial. Patients with pre-existing cerebral edema are at risk. Vigorous hydration leading to hyponatremia, seizures, and direct toxic effects of cisplatin on the blood-brain barrier and brain parenchyma may contribute.

\section{6.}

Steroid Induced CT Scan Changes in Patients with Recurrent Malignant Gliomas

\section{D.R. MACDONALD, J.G. CAIRNCROSS, J.H.W. PEXMAN and} R.K. COATES (London, Ontario)

Adrenocorticosteroid hormones (steroids) are known to decrease the enhancement, mass effect and edema associated with brain tumours. Since clinicians are relying increasingly on the computed tomographic (CT) scan to judge the response of malignant brain tumours to investigational treatments, it is crucial that the magnitude and time course of steroid induced CT scan changes be analyzed. It may be necessary to develop a strategy to distinguish CT scan improvement due to steroids from that due to other treatment modalities. This is especially relevant to the interpretation of phase II chemotherapy studies. At the time of recurrence many patients with malignant brain tumours are given steroids to control symptoms, followed immediately by investigational chemotherapy. The baseline CT scan documenting recurrence is performed prior to steroids. Treatment begins (i.e., steroids and antineoplastics) and the CT scan is repeated several months later. CT stability or improvement is attributed to chemotherapy without considering the role of steroids. To address this important clinical concern we have analyzed pre- and post-steroid CT scans in a small group of patients with recurrent malignant gliomas. Our methods and preliminary results are discussed.

37.

\section{Corticosteroid Toxicity in Neuro-Oncology Patients}

\section{D.E. WEISSMAN, D. DUFER and V. VOGEL (Baltimore, Maryland)}

The current trend towards higher doses and prolonged administration of corticosteroids among patients (pts) with brain tumours (BT) or epidural spinal cord compressions (ECC) has raised the potential for increasing toxicity. Records of all patients admitted to the Johns Hopkins Oncology Center (11/84-5/85) with either a BT or ECC, who received corticosteroids, were reviewed. 32 BT (primary-4, metastatic-28) and 27 ECC pts were identified, all having received dexamethasone. The mean daily dose and duration of administration was $16 \mathrm{mg}$ and 50 days in BT pts, $20 \mathrm{mg}$ and 26 days in ECC pts. Serious toxicity developed frequently including $20 \mathrm{pts}$ ( $34 \%$ ) with hyperglycemia of whom 8 required insulin, $20 \mathrm{pts}(34 \%)$ with infections including one death from candida sepsis, 15 pts (25\%) with peripheral edema, 11 pts (19\%) each with proximal myopathy and gastrointestinal problems including gastritis, Gl bleeding and sigmoid perforation, and 2 pts (3\%) with psychiatric events. 11 pts (19\%) required hospital admission 13 times specifically for diagnosis and/or management of steroid complications. Major toxicity was seen in only $2 / 59(3 \%)$ when the treatment duration was less than 21 days compared to $27 / 59(46 \%)$ when treatment was continued beyond three weeks. Treatment duration and total administered dose were predictive for major toxicity while age, sex and tumour histology had no predictive value. Corticosteroids cause significant morbidity when used in pts with brain tumours or epidural spinal cord compressions. To avoid excessive toxicity the duration of use and total administered dose should be kept to a minimum. Future research needs to be directed at finding non-toxic alternative drugs to treat these common oncologic problems.

38.

Brain Tumour Support Group as Adjunct to Psychosocial Services

\section{M.L. HEAD and N. STEELS (Vancouver, British Columbia)}

A support group for brain tumour patients, their families and friends has been operating since October, 1985. The primary focus of this group was to provide a meeting place for patients and their families to support one another in their common concerns. It was felt that this would offer direct benefits to our patients as well as make more effective and efficient use of interdisciplinary expertise.

The group population fluctuated between seven to fourteen persons; and a Doctor, Nurse and Social Worker attend as staff participants.

As more patients become aware, it is expected that the numbers will become much larger.

The process, patient attitudes and outcome of the group meetings will be discussed and conclusions drawn as to the merits of providing such a service in a clinical setting.

39.

Intracerebral Arterial Chemotherapy for Treatment of Advanced Childhood Brain Tumour - Report of a Child with Cerebral Primitive Neuroectodermal Tumour

\section{MACK, B. LUKE, D. STEWART, P. MCDONALD and E. VENTURERA (Ottawa, Ontario)}

Advanced brain tumour in children that have failed surgery, radiotherapy, and intravenous chemotherapy are uniformly fatal. Intracerebral arterial chemotherapy has been investigated as a more effective route of delivering chemotherapy in adult advanced brain tumours. No formal study has been done in children. We have attempted this route of treatment in a boy suffering from advanced primitive neuroectodermal tumour.

E.N., a 10-year-old boy with P.N.E.T. in the right cerebrum, relapsed 8 months after initial subtotal resection, radiotherapy and intravenous 
chemotherapy. Partial resection was again attempted. In view of rapid deterioration, we tried intra-arterial chemotherapy with VM-26, CisPlatinum and Carmustine infused by a femoral artery catheter to the internal carotid artery. Two injections over 4 week intervals were given. Tumour size was unchanged by CAT scan. Chemotherapy was changed to Mitomycin- $C, 3$ injections were given over $6-8$ week intervals. CAT scan showed decrease in tumour size with marked clinical improvement. Eight months later the tumour relapsed with pulmonary metastasis. He succumbed in 6 weeks.

The intra-arterial chemotherapy was well tolerated with surprisingly minimal complications and controlled progression of the tumour for 8 months. This treatment could be considered as an adjuvant therapy in advanced brain tumours in childhood.

40.

Treatment of Recurrent Malignant Gliomas with Blood-Brain Barrier Disruption and Intra-Arterial Chemotherapy

\section{J. ROZENTAL, A. LEVIN, P. TURSKI, L. HOUSTON, D. TRUMP, I. ROBINS and J. FINLAY (Madison, Wisconsin)}

Six patients with recurrent malignant gliomas underwent blood-brain barrier disruption (BBBD) with Renografin-76 and intra-arterial infusions of up to 3 cycles of $45-60 \mathrm{mg}$ of thio-TEPA until either progression or no response of the disease was evident. BBBD treatments were then followed with intra-arterial infusions of up to 9 cycles of $200 \mathrm{mg}$ of $\mathrm{BCNU}$ until progression of the disease. Survival and toxicities were assessed.

\begin{tabular}{|c|c|c|c|c|c|}
\hline Patient & Age & Therapy & & Survival & Tissue \\
\hline EB & 46 & $\mathrm{~T}-\mathrm{T} \times 3$ & BCNUX2 & 34 weeks & GBM \\
\hline DH & 41 & $\mathrm{~T}-\mathrm{T} \times 3$ & BCNUX6 & 69 weeks & Grlll \\
\hline RB & 33 & T-TX! & BCNUXI & 11 weeks & GBM \\
\hline $\mathbf{R R}$ & 42 & $T-T \times 3$ & BCNUXI & 11 weeks & GBM \\
\hline $\mathrm{PK}^{1}$ & 22 & $\mathrm{~T}-\mathrm{T} \times 2$ & $\mathrm{BCNU} \times 9^{2}$ & 43 weeks $^{3}$ & GBM \\
\hline MR & 55 & $\mathrm{~T}-\mathrm{T} \times 2$ & BCNUX5 & 23 weeks $^{3}$ & GBM \\
\hline $\mathbf{J K}^{\prime}$ & 24 & T-T $\times 3$ & & 8 weeks & GBM \\
\hline $\mathrm{JM}$ & 56 & $\mathrm{~T}-\mathrm{T} \times 3$ & BCNUXI & 10 weeks $^{3}$ & GBM \\
\hline
\end{tabular}

1 Patient failed 8 in -1 chemotherapy

2 Patients relapsed after 6 cycles of BCNU, so BCNU re-started 3 Patients still alive

Hematotoxicity (WBC less than 2500; PLT less than 100,000) was seen with 4 cycles of thio-TEPA and 2 of BCNU. One patient had ocular toxicity associated with a BCNU infusion. No liver, renal, or other blood chemistry abnormalities were discovered. BBBD with intra-arterial chemotherapy appears to be a promising therapeutic modality for treatment of malignant gliomas.

41.

Comparison of the Pharmacokinetics of Intra-Arterial and Intravenous BCNU Using Positron Emission Tomography

J.L. TYLER, Y.L. YAMAMOTO, M. DIKSIC, J. THÉRON, J.G. VILLEMURE, W.C. WORTHINGTON and W. FEINDEL (Montreal, Quebec)

Systemic chemotherapy for Grade III or IV gliomas has been disappointing in the past, however, recent reports have suggested that the intra-arterial administration of chemotherapeutic drugs can improve their efficacy. The incorporation of carbon-II, a positron emitter, into the structure of the cancer chemotherapeutic agent BCNU (1,3-bis(2chlorethyl)-nitrosourea) provides an opportunity to study the distribution and kinetics of this drug both in normal brain tissue and in brain tumours. BCNU is especially well-suited for the intra-arterial route, because of its short biological half-life, its high lipid solubility, and its avid uptake by malignant gliomas.

The objectives of this research are two-fold; the establishment of the pharmacokinetics of BCNU using positron emission tomography, and the investigation of possible increased efficacy of this drug when administered by a super-selective intra-arterial route. In order to investigate the pharmacokinetics of " $\mathrm{C}-\mathrm{BCNU}$, sequential scans obtained after the intravenous injection of "C-BCNU are compared with studies obtained after the injection of this compound through super-selective intra-arterial catheter situated in the main tumour feeding vessel. Timeactivity curves for "-C-radioactivity in tumour and in normal brain were generated; these were analyzed by stripping off the long slow component, and holding this constant, analyzing the initial rapid component with a non-linear least squares fit. Preliminary results in twelve cases demonstrate two distinct components of the "C-BCNU timeactivity curve; the first being proportional to blood flow, and the second representing " $\mathrm{C}$ - $\mathrm{BCNU}$ uptake in tissues. Tissue radioactivity levels demonstrate that only mild increases in tumour concentration relative to normal brain were achieved by intravenous injection of "C-BCNU, while intra-arterial injection increased the tumour concentration of " $\mathrm{C}$-activity up to 380 times that of the normal brain. Comparison of the heights of the peak activity in tumour and normal brain after bolus intravenous injection was used to approximate relative tumour blood flow. The concentration of " $\mathrm{C}$-radioactivity in tumour was found to be independent of blood flow.

In two cases, dramatic tumour regression was seen within three weeks of super-selective intra-arterial BCNU chemotherapy. After examining the 10 minute tissue activity after intra-arterial versus intravenous injection, it was noted that the cases having the highest 10 minute values were those that were subsequently found to have the most dramatic tumour response to super-selective intra-arterial BCNU chemotherapy. This response was found to be independent of relative tumour blood flow.

The studies to date have shown significant differences between the intra-arterial and intravenous routes of " $\mathrm{C}$-BCNU injection, both in increasing tumour concentrations of $\mathrm{BCNU}$, and in sparing normal tissue. There is some suggestion that the degree of initial metabolic trapping of "C-BCNU may prove useful in predicting a favourable clinical response.

42.

Theoretical Comparison of Intra-Arterial and Intravenous Infusion of $\mathrm{BCNU}$

\section{DIKSIC (Montreal, Quebec)}

Intra-arterial (IA) and intravenous (IV) infusion of 1.3-bis-(2-chlorethyl)nitrosourea (BCNU) were compared by a one- and two-compartment biological model, respectively. The uptake into the brain of BCNU. a lipid soluble drug, is greatly influenced by the blood-brain partition coefficient (p). Since the half-life of BCNU is only $-20 \mathrm{~min}$ in plasma and probably close to that in tissue, a high tissue concentration for a prolonged period would be necessary for the drug to be therapeutically beneficial. We found that fragments of " $\mathrm{C}$-labelled BCNU can be visualized $\sim 15$ min after injection.

Three sets of partition coefficients were investigated. When the partition coefficient changes from 50 to 10 , the absolute amount of the drug decomposed in the brain is reduced by half.

Since the half-life of BCNU in an injection solution is between 100 and $150 \mathrm{~min}$, the duration of the infusion was also investigated. The 
Three sets of partition coefficients were investigated. When the partition coefficient changes from 50 to 10 , the absolute amount of the drug decomposed in the brain is reduced by half.

Since the half-life of BCNU in an injection solution is between 100 and $150 \mathrm{~min}$, the duration of the infusion was also investigated. The simulation indicates that extending the duration of the infusions, IV or $1 \mathrm{~A}$, to more than the half-life of BCNU in the syringe, had no beneficial effect. The reason for this is combined effect of the short half-life of the drug in plasma as well as decomposition in the syringe.

In general. IA-infusion delivers 2 to 10 times more drug to the brain than does IV-infusion. The reasons for this difference and the spread are the half-life and other biological parameters which define body distribution of $\mathrm{BCNU}$ and decomposition of tissue $\mathrm{BCNU}$. The simulation also shows that increasing the tissue $\mathrm{pH}$ to about $0.4 \mathrm{pH}$ units would increase the decomposed $\mathrm{BCNU}$ in that tissue by $\sim 1.8$. This is in agreement with data we obtained with PET and "C-labelled BCNU.

This work has been supported in part by the Medical Research Council of Canada (SP-5), an NIH grant (No. 1-ROI-NS-22230-01NENA-T), and the Killam Memorial Scholarship Fund of the MNI.
43.

Superselective Cerebral Artery Infusion of BCNU in High Grade Glioma

J.F. EMRICH, J.-G. VILLEMURE, R. LEBLANC, C. WORTHINGTON, D. MELANSON, J. THÉRON, S.FONTAINE and K.M. VILLEMURE (Montreal, Quebec)

With recent advances in radiological techniques, intraarterial chemotherapy for high grade gliomas is becoming more widespread. This form of therapy has resulted in unique problems related to technique and drug toxicity. We present our experience with supra-ophthalmic internal carotid BCNU infusions with an analysis of complications. Forty-nine catheterizations have been performed on twenty-four patients and forty-one treatments have been given. $75 \%$ of patients responded to this therapy. Complications were analyzed and categorized as follows:

1. Complications due to technique

a) Catheter-induced vasospasm $\quad 12 \%$

b) Thrombo-embolism $0 \%$

2. Complications due to drug toxicity

a) Systemic toxicity $\quad 0 \%$

b) Ocular toxicity $5 \%$

c) Early cerebral toxicity $\quad 17 \%$

d) Late cerebral toxicity $\quad 8 \%$

Supra-ophthalmic infusion, avoidance of alcohol diluent and administration of a lower dose of $\mathrm{BCNU}$ are effective in reducing systemic, ocular and cerebral toxicity while still effecting a reduction or stabilization of tumour size. 